\title{
Saúde sexual e reprodutiva de homens transgêneros e mulheres homoafetivas:
}

\section{Revisão Integrativa}

\author{
Sexual and reproductive health of transgender men and homoaffective women: Integrative Review \\ Salud sexual y reproductiva de hombres transgénero y mujeres homoafectivas: Revisión integrativa
}

Pâmela Mendes Arruda

ORCID: https://orcid.org/0000-0002-1661-8994 Universidade Federal do Ceará, Brasil

E-mail: pam_mendesarruda@yahoo.com.br

Manuela Gondim Lima Oliveira

ORCID: https://orcid.org/0000-0001-6041-2486 Universidade Federal do Ceará, Brasil E-mail: manugondimlima@gmail.com

Isabela Aragão Colares

ORCID: https://orcid.org/0000-0002-9503-1103 Universidade Federal do Ceará, Brasil E-mail: Isabelaacolares@alu.ufc.br

Débora Fernandes Brito

ORCID: https://orcid.org/0000-0001-6248-8054 Universidade Federal do Ceará, Brasil E-mail: deborabritto@hotmail.com

Raquel Autran Coelho Peixoto

ORCID: https://orcid.org/0000-0002-2998-2779 Universidade Federal do Ceará, Brasil E-mail: raquelautrancp@gmail.com

\begin{abstract}
Resumo
Objetivo: Há uma compreensão limitada sobre peculiaridades no cuidado à saúde de minorias sexuais e de gênero (MSG). Este estudo objetivou discutir as necessidades clínicas e principais barreiras da população transgênero e homoafetiva no cenário da ginecologia. Metodologia: Foi realizada uma revisão integrativa da literatura, das bases de dados on-line: MEDLINE, SCIELO e LILACS dos últimos cinco anos, utilizando-se a pergunta norteadora: "Quais as recomendações atuais para o cuidado à saúde em ginecologia para homens transgênero e mulheres homoafetivas, quanto à proteção à saúde e prevenção de doenças?” Resultados: Ao final das buscas, 13 publicações atenderam aos critérios de elegibilidade e foram selecionadas para compor o estudo. Foram revisadas as recomendações específicas para promoção à saúde de homens transgênero e mulheres homoafetivas, quanto à consulta ginecológica, prevenção de câncer, ISTs e planejamento reprodutivo. O conhecimento das necessidades de saúde permite desenvolver uma prática acolhedora e inclusiva para as pessoas de MSGs. O aumento da educação e treinamento entre provedores e profissionais de saúde e melhorias gerais no sentido de compreender as barreiras à triagem de saúde e à captação de recursos de saúde podem reduzir algumas disparidades. Conclusão: Os profissionais de saúde devem se familiarizar com as necessidades únicas de atenção à saúde de pessoas que se identificam como homens transgêneros e mulheres homoafetivas. Diversos determinantes sociais podem influenciar o atendimento ginecológico de MSGs, podendo gerar disparidades de saúde nesses grupos marginalizados.
\end{abstract}

Palavras-chave: Minorias sexuais e de gênero; Pessoas transgênero; Homossexualidade; Ginecologia.

\begin{abstract}
Objective: There is limited understanding of peculiarities in the health care of sexual and gender minorities (SGM). This study aimed at needs such as clinical and main barriers of the population and homosexuals in the scenario of transformation. Methodology: An integrative literature review was carried out, using as online databases: MEDLINE, VHL and LILACS of the last 5 years, using the guiding question: "What are the current ones for health care in gynecology for transgender and gay men, to health protection and disease prevention?" Results: At the end of the searches, 13 publications met the eligibility criteria and were selected to compose the study. They were reviewed as specific for promoting the health of transgender men and homosexual women, regarding gynecological consultation, cancer and STI prevention and reproductive planning. Knowledge of health needs allows the development of a welcoming and inclusive practice for SGMs. Education education and training among health providers and professionals can improve the sense of understanding how health screening and health fundraising can increase
\end{abstract}


disparities. Conclusion: Health professionals should become familiar with the unique health care needs of people who identify as transgender men and gay women. Social determinants can influence gynecological care for SGMs, which can generate disparities among different marginalized groups.

Keywords: Sexual and gender minorities; Transgender person; Homosexuality; Gynecology.

\section{Resumen}

Objetivo: Existe una comprensión limitada de las peculiaridades en el cuidado de la salud de las minorías sexuales y de género (MSG). Este estudio apuntó a necesidades como clínica y principales barreras de la población y homosexuales en el escenario de transformación. Metodología: Se realizó una revisión integrativa de la literatura, utilizando como bases de datos en línea: MEDLINE, BVS y LILACS de los últimos 5 años, utilizando como pregunta guía: “¿Cuáles son las actuales para la atención en salud en ginecología para hombres transgénero y gays, para la salud protección y prevención de enfermedades? Resultados: Al final de las búsquedas, 13 publicaciones cumplieron con los criterios de elegibilidad y fueron seleccionadas para componer el estudio. Fueron revisados como específicos para la promoción de la salud de hombres transgénero y mujeres homosexuales, en cuanto a consulta ginecológica, prevención de cáncer e ITS y planificación reproductiva. El conocimiento de las necesidades de salud permite desarrollar una práctica acogedora e incluyente para las MSGs. La educación, la educación y la capacitación entre los proveedores y profesionales de la salud pueden mejorar la comprensión de cómo las pruebas de detección y la recaudación de fondos para la salud pueden aumentar las disparidades. Conclusión: Los profesionales de la salud deben familiarizarse con las necesidades únicas de atención médica de las personas que se identifican como hombres transgénero y mujeres homosexuales. Los determinantes sociales pueden influir en la atención ginecológica de las MSGs, lo que puede generar disparidades entre los diferentes grupos marginados.

Palabras clave: Minorías sexuales y de género; Personas transgénero; Homosexualidad; Ginecología.

\section{Introdução}

Na perspectiva binária e heterossexual, masculinidade(s) e feminilidade(s) são compreendidas como a expressão cultural da diferença natural (biológica) dos sexos e que, em decorrência disso, todos os elementos constitutivos ou de expressão dos sujeitos estão condicionados a essa determinação (Bento, 2017). Desta forma, a lógica hegemônica de sexo biológico como fator que determina a identidade de gênero torna estranho qualquer um que fuja da lógica binária e dicotômica (Popadiuk et al., 2017).

Uma pessoa transgênero é aquela que não se identifica com o gênero que lhe foi atribuído ao nascer. Os termos "transgeneridade" / "transgênero" (ou a abreviação "Trans") fazem referência à identidade de gênero de pessoas que não se identificam com o gênero designado ao nascer, utilizando o termo como "conceito guarda-chuva" - dentro do qual cabem muitas diferentes identidades (Bento, 2017). Ocorre situação de gênero fluido quando a identidade pessoal do indivíduo muda de expressão masculina para feminina para não-binária, podendo variar de dia para dia, fazendo com que se inicie e interrompa o tratamento hormonal e potencialmente aumentando riscos médicos. É importante validar a identidade desses indivíduos e discutir situações peculiares às suas vivências (Nisly et al., 2018).

A orientação sexual não foi conclusivamente determinada por fatores específicos, e o momento do surgimento, reconhecimento e expressão da orientação sexual de uma pessoa varia entre os indivíduos. Para muitas mulheres, a orientação sexual segue um continuum em que uma mulher pode não ser exclusivamente heterossexual ou homossexual (ACOG, 2021). Os termos homossexual e homoafetivo comumente são encarados como sinônimos, mas homoafetivo descreve a complexidade e a multiplicidade de relações afetivas e/ou sexuais entre pessoas do mesmo sexo/gênero, enquanto homossexual conota também os aspectos emocionais e afetivos envolvidos na relação amorosa entre pessoas do mesmo sexo/gênero (Reis, 2018).

“Todo indivíduo possui direito de igualdade e liberdade no exercício da sexualidade; estes são: autonomia sexual, integridade sexual e segurança corpórea, privacidade, prazer, expressão, associação, escolhas reprodutivas e informação sexual livre de discriminação" (Ávila, 2003). Apesar de tais prerrogativas, no atendimento básico de indivíduos transgêneros e homoafetivos, essa parcela vulnerável da população ainda enfrenta diversos constrangimentos, como episódios transfóbicos e homofóbicos por profissionais ou demais usuários do serviço de saúde, incluindo o não uso do nome social e pronomes 
adequados em cadastros e prontuários, o que reduz a adesão aos sistemas de saúde.

No Brasil, ainda se fazem presentes manifestações de homofobia, transfobia e o preconceito inclusive nos serviços de saúde, sustentando e agravando o deficitário atendimento a ser ofertado no Sistema Único de Saúde, indo na contramão das políticas de cuidado humanizado preconizadas (Lovison et al., 2019). Nesse contexto, a recusa em considerar as especificidades em saúde dos transgêneros prejudica o reconhecimento das variações sociais, subjetivas e biológicas do ser humano (Lionço, 2009).

No que diz respeito a essas demandas e individualidades, a Carta dos Direitos dos Usuários da Saúde (Brasil, 2012) assegura não só o preenchimento do nome social nos documentos de identificação do SUS (Cartão Nacional de Saúde/CNS) como também explicita o direito ao cuidado, ao tratamento e ao atendimento no âmbito do SUS, livres de discriminação por orientação sexual e identidade de gênero, vetando o uso de formas desrespeitosas e preconceituosas no atendimento.

Sob esse viés, as minorias sexuais e de gênero (MSG) são as que mais enfrentam dificuldades ao buscarem atendimentos nos serviços públicos de saúde - não só quando reivindicam serviços especializados, como o processo transexualizador, mas em diversas outras ocasiões nas quais buscam atendimento - pela enérgica trans/travestifobia que sofrem atrelada à discriminação por outros marcadores sociais - como pobreza, raça/cor, aparência física - e pela escassez de serviços de saúde específicos (Mello et al., 2011).

É dentro dessa perspectiva, com a finalidade de discutir as possibilidades de ampliação de um conhecimento transdisciplinar que permita auxiliar profissionais no cuidado adequado à saúde das MSG, que se justificou o estudo das práticas de atenção à saúde sexual e reprodutiva de transgêneros e homossexuais. Considera-se que essas relações sociais de desigualdade de gênero necessitam de um olhar holístico, centrado na promoção da saúde, com políticas públicas que assegurem qualidade na assistência, mas também considerando as facetas discriminatórias possivelmente presentes na atenção à saúde de dessa população. Esta revisão se concentra nas barreiras e necessidades clínicas da população transgênero e homoafetiva no cenário da ginecologia.

\section{Metodologia}

Foi realizada uma revisão integrativa da literatura teórica e empírica. Trata-se de uma abordagem metodológica mais ampla que permite a inclusão de estudos experimentais e não experimentais para obter uma melhor compreensão do fenômeno analisado. Seu impacto ocorre não somente pelo desenvolvimento de políticas, protocolos e procedimentos, mas também pelo pensamento crítico de que a prática diária necessita (Souza et al., 2010)

Foram utilizadas as seguintes etapas para sua elaboração: (1) delimitação do tema e construção da pergunta norteadora da pesquisa; (2) levantamento das publicações nas bases de dados selecionadas; (3) classificação e análise das informações achadas em cada manuscrito; (4) análise dos estudos escolhidos; (5) apresentação dos resultados encontrados e (6) inclusão, análise crítica dos achados e síntese da revisão da literatura (Souza et al., 2010).

A pergunta norteadora utilizada para coletar informações e delimitar os resultados foi: "Quais as recomendações atuais para o cuidado à saúde em ginecologia para pacientes de MSG, quanto à proteção à saúde e prevenção de doenças?"

Foi realizada amostragem na literatura de maneira ampliada e diversa, no período de agosto a novembro de 2021, utilizando-se os seguintes descritores: ((MINORIAS SEXUAIS E DE GÊNERO) OR (TRANSGÊNEROS) AND (HOMOSSEX*)) AND ((GINECOLOGIA) AND (SAÚDE)). Utilizaram-se as seguintes bases de dados on-line: Biblioteca Virtual em Saúde (BVS), nas Bases de Dados da Literatura Latino-Americana em Ciências de Saúde (LILACS) e Base de Dados Bibliográficos da Biblioteca Nacional de Medicina dos Estados Unidos da América (MEDLINE), dos últimos 5 anos.

$\mathrm{Na}$ fase da coleta, utilizou-se um instrumento elaborado para garantir que fossem extraídos todos os dados relevantes, 
a fim de diminuir erros de transcrição, garantir precisão de informações e servir como registro (Souza et al., 2010) Utilizaramse alguns critérios de inclusão: artigos completos, com assunto principal correspondendo em alguma medida à pergunta norteadora, independentemente do tipo de estudo, do idioma. Foram excluídos os trabalhos indisponíveis para acesso na íntegra, duplicados, e artigos que estavam fora da delimitação temporal pré-estabelecida ou que não respeitavam objetivo do estudo e a pergunta norteadora.

Inicialmente, foi realizada a leitura dos títulos, em um segundo momento, a leitura dos resumos e posteriormente do artigo completo. O processo de seleção dos artigos, bem como os resultados, está apresentado na Figura 1.

Figura 1: Fluxograma PRISMA de seleção dos estudos que constituíram a amostra.
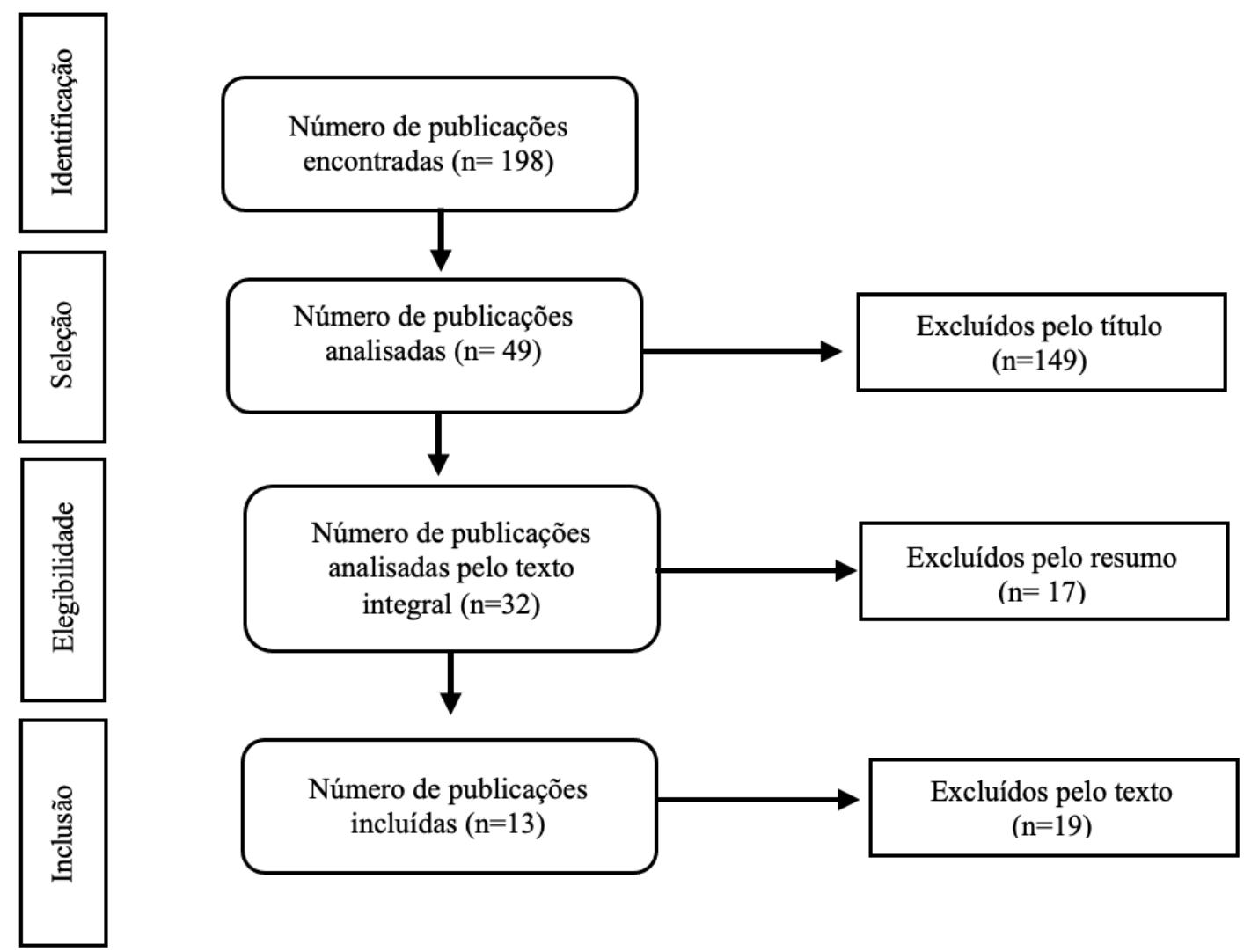

Fonte: Autores.

A análise crítica dos estudos selecionados e a síntese dos dados bibliográficos foram realizadas de forma descritiva, agrupados por tópicos pertinentes à saúde em Ginecologia de MSGs, com a intenção de colaborar com o processo de busca da temática principal deste trabalho.

$\mathrm{Na}$ etapa de discussão dos resultados, realizou-se síntese das evidências a partir da análise dos artigos com base na pergunta norteadora, de modo a tornar possível a delimitação de estudos e o apontamento de protocolos de recomendações.

\section{Resultados e Discussão}

Ao final das buscas, 13 publicações atenderam aos critérios de elegibilidade e foram selecionadas para compor o estudo. Os estudos foram desenvolvidos nos Estados Unidos $(n=11)$, Argentina $(n=01)$, e Brasil ( $n=1)$. Houve somente um estudo brasileiro que inclui aspectos da pergunta norteadora, tratando especificamente de práticas sexuais e cuidado em saúde 
de mulheres que fazem sexo com mulheres (Rufino et al., 2018). No Quadro 1 é possível observar a síntese de algumas informações referentes aos estudos incluídos na revisão integrativa.

Quadro 1: Distribuição dos artigos selecionados, Fortaleza, 2021.

\begin{tabular}{|c|c|c|c|c|c|}
\hline $\mathrm{N}^{\circ}$ & Título & Autores & Objetivo & Metodologia & $\begin{array}{l}\text { País da } \\
\text { publicação }\end{array}$ \\
\hline 1 & $\begin{array}{l}\text { The } \mathrm{Ob} / \mathrm{Gyn} \text { and the } \\
\text { transgender patient. }\end{array}$ & Jarin J.(2019) & $\begin{array}{l}\text { Ajudar os ginecologistas a } \\
\text { reconhecer seu papel no cuidado de } \\
\text { pacientes transgêneros. }\end{array}$ & $\begin{array}{l}\text { Revisão } \\
\text { narrativa }\end{array}$ & EUA \\
\hline 2 & $\begin{array}{l}\text { Transgender patients: } \\
\text { considerations for routine } \\
\text { gynecologic care and } \\
\text { cancer screening. }\end{array}$ & $\begin{array}{l}\text { Labanca T, Mañero I, } \\
\text { Pannunzio M. (2020) }\end{array}$ & $\begin{array}{l}\text { Resumir as recomendações atuais } \\
\text { para ginecologistas em relação a } \\
\text { câncer em pessoas transgênero }\end{array}$ & $\begin{array}{l}\text { Revisão } \\
\text { narrativa }\end{array}$ & Argentina \\
\hline 3 & $\begin{array}{l}\text { Do Sexual Minorities } \\
\text { Receive Appropriate } \\
\text { Sexual and Reproductive } \\
\text { Health Care and } \\
\text { Counseling? }\end{array}$ & $\begin{array}{l}\text { Everett BG, Higgins } \\
\text { JA, Haider S, } \\
\text { Carpenter E. (2019) }\end{array}$ & $\begin{array}{l}\text { Investigar a saúde sexual e } \\
\text { reprodutiva entre as mulheres de } \\
\text { minorias sexuais }\end{array}$ & Quantitativo & EUA \\
\hline 4 & $\begin{array}{l}\text { Care and Cancer } \\
\text { Screening of the } \\
\text { Transgender Population. }\end{array}$ & $\begin{array}{l}\text { Puechl AM, Russell } \\
\text { K, Gray BA (2019) }\end{array}$ & $\begin{array}{l}\text { Descrever práticas apropriadas de } \\
\text { triagem de câncer e considerações } \\
\text { importantes sobre cuidados para o } \\
\text { médico da atenção primária e o } \\
\text { ginecologista que cuida de } \\
\text { individuos transgêneros }\end{array}$ & $\begin{array}{l}\text { Revisão } \\
\text { narrativa }\end{array}$ & EUA \\
\hline 5 & $\begin{array}{l}\text { Routine Screening for } \\
\text { Transgender and Gender } \\
\text { Diverse Adults Taking } \\
\text { Gender-Affirming } \\
\text { Hormone Therapy: a } \\
\text { Narrative Review. }\end{array}$ & $\begin{array}{l}\text { Iwamoto SJ, } \\
\text { Grimstad F, Irwig } \\
\text { MS, Rothman MS. } \\
(2021)\end{array}$ & $\begin{array}{l}\text { Ajudar os profissionais de saúde no } \\
\text { aconselhamento e triagem de } \\
\text { pacientes transgêneros e com } \\
\text { gêneros diversos }\end{array}$ & $\begin{array}{l}\text { Revisão } \\
\text { narrativa }\end{array}$ & EUA \\
\hline 6 & $\begin{array}{l}\text { Clinical needs for } \\
\text { transgender men in the } \\
\text { gynecologic oncology } \\
\text { setting }\end{array}$ & $\begin{array}{l}\text { Stenzel AE, Moysich } \\
\text { KB, Ferrando CA, } \\
\text { Starbuck KD. (2020) }\end{array}$ & $\begin{array}{l}\text { Discutir as necessidades clínicas de } \\
\text { homens transgêneros no cenário da } \\
\text { oncologia ginecológica }\end{array}$ & $\begin{array}{l}\text { Revisão } \\
\text { narrativa }\end{array}$ & EUA \\
\hline 7 & $\begin{array}{l}\text { Health Care for } \\
\text { Transgender and Gender } \\
\text { Diverse Individuals: } \\
\text { ACOG Committee } \\
\text { Opinion, Number } 823\end{array}$ & ACOG. (2021) & $\begin{array}{l}\text { Fornecer informações clínicas sobre } \\
\text { hormonioterapia e cuidados } \\
\text { preventivos para pessoas } \\
\text { transgênero e de gênero diverso }\end{array}$ & $\begin{array}{l}\text { Revisão } \\
\text { narrativa }\end{array}$ & EUA \\
\hline 8 & $\begin{array}{l}\text { Addressing the Needs of } \\
\text { Transgender Patients: } \\
\text { How Gynecologists Can } \\
\text { Partner in Their Care }\end{array}$ & $\begin{array}{l}\text { Dendrinos ML, } \\
\text { Budrys NM, Sangha } \\
\text { R. (2019) }\end{array}$ & $\begin{array}{l}\text { Identificar os principais problemas } \\
\text { ginecológicos enfrentados por } \\
\text { pacientes transgêneros }\end{array}$ & $\begin{array}{l}\text { Revisão } \\
\text { narrativa }\end{array}$ & EUA \\
\hline 9 & $\begin{array}{l}\text { Clinical Care of Lesbian } \\
\text { and Bisexual Women for } \\
\text { the Obstetrician } \\
\text { Gynecologist. }\end{array}$ & $\begin{array}{l}\text { McCune KC, } \\
\text { Imborek KL.(2018) }\end{array}$ & $\begin{array}{l}\text { Discutir as disparidades de cuidados } \\
\text { de saúde, bem como } \\
\text { comportamentos em mulheres de } \\
\text { minorias sexuais }\end{array}$ & $\begin{array}{l}\text { Revisão } \\
\text { narrativa }\end{array}$ & EUA \\
\hline 10 & $\begin{array}{l}\text { Sexual practices and } \\
\text { health care of women who } \\
\text { have sex with women: } \\
2013-2014 \text {. }\end{array}$ & $\begin{array}{l}\text { Rufino AC, Madeiro } \\
\text { A, Trinidad A, Santos } \\
\text { R, Freitas I. (2018) }\end{array}$ & $\begin{array}{l}\text { Descrever práticas sexuais e } \\
\text { cuidados com a saúde de mulheres } \\
\text { que fazem sexo com mulheres }\end{array}$ & Quantitativo & Brasil \\
\hline 11 & $\begin{array}{l}\text { Reproductive Health Care } \\
\text { Priorities and Barriers to } \\
\text { Effective Care for LGBTQ } \\
\text { People Assigned Female at } \\
\text { Birth: A Qualitative Study. }\end{array}$ & $\begin{array}{l}\text { Wingo E, Ingraham } \\
\text { N, Roberts SCM. } \\
(2018)\end{array}$ & $\begin{array}{l}\text { Identificar prioridades de saúde } \\
\text { reprodutiva de indivíduos LGBTQ }\end{array}$ & $\begin{array}{l}\text { Revisão } \\
\text { narrativa }\end{array}$ & EUA \\
\hline 12 & $\begin{array}{l}\text { Preventive Health for } \\
\text { Transgender Men and } \\
\text { Women. }\end{array}$ & $\begin{array}{l}\text { Imborek KL, Graf } \\
\text { EM, McCune K. } \\
(2017)\end{array}$ & $\begin{array}{l}\text { Revisar e sugerir melhores práticas } \\
\text { para otimizar a saúde preventiva da } \\
\text { população transgênero. }\end{array}$ & $\begin{array}{l}\text { Revisão } \\
\text { narrativa }\end{array}$ & EUA \\
\hline 13 & $\begin{array}{l}\text { Understanding } \\
\text { Transgender Men's } \\
\text { Experiences with and } \\
\text { Preferences for Cervical } \\
\text { Cancer Screening: A } \\
\text { Rapid Assessment Survey. }\end{array}$ & $\begin{array}{l}\text { Seay J, Ranck A, } \\
\text { Weiss R, Salgado C, } \\
\text { Fein L, Kobetz E. } \\
(2017)\end{array}$ & $\begin{array}{l}\text { Compreender experiências e } \\
\text { preferências para o rastreamento do } \\
\text { câncer do colo do útero entre } \\
\text { homens transgêneros }\end{array}$ & Quantitativo & EUA \\
\hline
\end{tabular}


Há poucos estudos com detalhamento sobre o número de pessoas transexuais e travestis no Brasil, mas pesquisas realizadas em diversas populações mundiais concluíram que correspondem a $0,5 \%$ a $1,3 \%$ da população geral (Winter et al., 2016). Indivíduos brasileiros com qualquer diversidade de gênero parecem representar cerca de $2 \%$ da população adulta do país e estão distribuídos homogeneamente pelas 5 regiões (Spizzirri et al., 2021).

Embora as estatísticas de prevalência variem nos Estados Unidos, dados apontam que 1,1\% e 3,5\% das mulheres se identificam como lésbicas ou bissexuais, respectivamente (Chandra et al., 2013). Cerca de 0,6\% da população adulta americana se identifica como transgênero. Um quarto dos adultos transgêneros passaram por cirurgias de afirmação de gênero e outros 43\% procuram se submeter a qualquer uma das várias cirurgias de afirmação de gênero (James et al., 2016).

\section{Consulta Ginecológica}

As MSG devem receber cuidados abrangentes à sua saúde, incluindo prevenção de doenças cardiovasculares, obesidade, câncer e infecções sexualmente transmissíveis (ISTs), além de atenção à saúde reprodutiva. As necessidades de cuidados em saúde reprodutiva nessa população incluem necessidades semelhantes a grupos cisgênero e heterossexuais (como tratamento da síndrome dos ovários policísticos, menstruação irregular e contracepção), bem como necessidades únicas (como histerectomias de afirmação de gênero e assistência à fertilidade) e desafios na busca de cuidados (Wingo et al., 2018).

Devido ao medo do constrangimento, humilhação e violência nas salas de espera ou até mesmo durante o atendimento ginecológico, homens transgêneros que não foram submetidos a cirurgia de redesignação sexual demonstram pouca adesão a essa área da saúde. Tal conjuntura preocupante pode ser concretizada pelo fato de homens trans terem seus testes de Papanicolau menos atualizados em relação a mulheres cisgênero (Stewart et al., 2020). Homens transgêneros, assim como mulheres cisgêneros, permanecem em risco de malignidades ginecológicas, mas são uma população carente de atenção à saúde sexual e reprodutiva. Segundo Coleman et al. (2012), o acolhimento inicial do usuário no serviço, seja ele quem for, mas em especial as MSG, é fator determinante e deve ser foco de capacitações.

Vale ressaltar que os pacientes devem ser chamados pelo nome de preferência. É importante que os homens transgêneros não sejam tratados como "mulheres cisgêneros". É útil usar terminologia neutra em termos de gênero ou perguntar ao paciente que linguagem ele usa para se referir às partes do corpo, incluindo a genitália. Pode ser preferível, por exemplo, dizer que fará "exame de tórax" em vez de dizer "exame de mama" (Nisly et al., 2018)

As terapias de transição ou de "afirmação de gênero" incluem terapia hormonal e/ou procedimentos cirúrgicos que ajudam os indivíduos a alinhar seus corpos com seu senso interno, mas nem todos os membros da comunidade transgênero passarão por intervenção farmacológica ou cirúrgica. Algumas técnicas cirúrgicas disponíveis para transformação corporal logicamente afetam a fertilidade, bem como o uso de hormônios para transformação corporal (Hembree et al., 2017). Dentre as intervenções cirúrgicas, os homens transgêneros podem ser submetidos a mastectomia, histerectomia (com ou sem salpingooforectomia bilateral), vaginectomia e cirurgias reconstrutivas para criação de genitália externa. Dada a sua experiência cirúrgica e experiência na gestão hormonal, os ginecologistas-obstetras estão numa posição única para prestar cuidados de saúde de qualidade a esta população (Jarin, 2019).

$\mathrm{O}$ uso prolongado de estrógeno, assim como o uso de antiandógeno, reduz a qualidade e a concentração de espermatozoides no sêmen, enquanto o uso prolongado de testosterona tende a causar atrofia endometrial, anovulação, e mesmo osteoporose (Iwamoto et al., 2021). A hormonização em pessoas transexuais e travestis, por outro lado, não pode ser considerada como contracepção efetiva (De Hoo et al., 2016). A terapia hormonal baseia-se principalmente em testosterona exógena, o que ajuda os pacientes a desenvolver características físicas masculinas (Coleman et al., 2012; Obedin-Maliver \& de Haan, 2017; White Hughto et al., 2017). A extensão das mudanças adquiridas através da terapia depende da dosagem e da duração do uso, com possível aumento de pelos faciais/corporais, alterações vocais e na aparência genital e interrupção da 
menstruação. As opções contendo estrogênio geralmente são evitadas. Opções de progesterona podem ser utilizados para ajudar a interromper a menstruação, caso necessário (Obedin-Maliver \& de Haan, 2017).

Ao buscarem suprir o desconforto de apresentarem um corpo não desejado por eles, pessoas transgênero procuram diariamente meios de reposição hormonal. No entanto, existe certa demora no acesso a esse processo, o que faz com que a maioria das pessoas transgênero que chegam ao médico já tenha feito alguma tentativa de transição por meio da automedicação, geralmente por métodos encontrados na internet ou por indicação de amigos (Arán et al., 2009). Em paralelo a esses dados, o medo de sofrer algum tipo de preconceito e discriminação ao buscarem o atendimento ginecológico ou agendarem uma consulta com um endocrinologista contribuem para essas práticas de automedicação, trazendo inúmeros risco a saúde do paciente (Fernandez et al., 2016). Esse contexto de desinformação contribui para a falta de orientação sobre as consequências da terapia de reposição hormonal na fertilidade dos indivíduos e na anticoncepção.

\section{Prevenção de Câncer Ginecológico}

Houve algum esforço na tentativa de identificar a prevalência de malignidades em pacientes de MSG, mas a evidência é severamente limitada (Urban et al., 2011). Atualmente, não existem diretrizes específicas nas sociedades endócrinas ou ginecológicas delineando a vigilância sugerida para malignidades hormonalmente sensíveis em homens transgêneros em terapia hormonal de afirmação de gênero. Geralmente, a vigilância de rotina apropriada para a idade é recomendada, mas dados adicionais nesta população são necessários para avaliar mais completamente o risco e as variações na apresentação (Stenzel et al., 2020)

A terapia com testosterona frequentemente induz amenorreia e aumenta a virilização, no entanto, faltam dados de segurança a longo prazo, pelo potencial mecanismo de aromatização para estrogênio. Isso poderia elevar o risco de cânceres hormonalmente responsivos, como o câncer de endométrio tipo I. Com base nos dados limitados, a testosterona não parece aumentar a hiperplasia endometrial em populações transmasculinas pré ou pós-menopáusicas; em muitos casos a testosterona induz atrofia semelhante ao que é visto em pacientes do sexo feminino cisgênero. Em uma revisão retrospectiva multiinstitucional de 94 pacientes em terapia de afirmação de gênero com testosterona posteriormente submetidas à histerectomia, somente 1 paciente $(1,2 \%)$ apresentava hiperplasia endometrial sem atipia, e 24,5\% apresentavam histopatologia atrófica. Além disso, a duração da terapia com testosterona não foi associada à espessura da proliferação endometrial. Não é possível determinar como avaliar o risco de forma mais eficaz; no entanto, ainda é importante que os profissionais revisem os sintomas preocupantes, como sangramento irregular ou pós-menopausa, com pacientes em uso de terapia com testosterona (Urban et al., 2011; Puechl et al, 2019; Grimstad et al., 2019; Stenzel et al., 2020). A testosterona também foi hipotetizada para contribuir para a carcinogênese ovariana em homens transgêneros, principalmente para os subtipos endometrioide e mucinoso, mas com base em estudos limitados (Urban et al., 2011).

Quanto ao rastreamento de câncer de mama, a mamografia pode apresentar benefícios semelhantes aos das mulheres cisgênero para homens transgênero que não tiveram suas glândulas mamárias removidas e para mulheres trans em uso de hormônios. A oferta deve incluir informações de benefícios e riscos, como $0,1 \%$ de detecção precoce efetiva no rastreamento para população com baixo risco para câncer mamário (Gøtzsche \& Jørgensen, 2013). Um estudo recente que investigou os riscos de câncer de mama em indivíduos transgêneros utilizando terapia hormonal encontrou um risco diminuído em homens transgêneros em comparação com homens e mulheres cisgênero. O estudo concluiu que as diretrizes de triagem de câncer de mama usadas para indivíduos cisgêneros são suficientes para pessoas transgênero que utilizam terapia hormonal.

Há uma escassez de evidências confiáveis sobre o rastreamento do câncer de mama para homens transgêneros submetidos à mastectomia bilateral resultando em recomendações variadas (Puechl et al., 2019). Homens transgêneros que se submeteram à mastectomia não precisam mais de mamografias, no entanto, ainda pode haver valor em autoexames regulares 
das mamas, pois o câncer de mama ainda foi relatado nesse cenário (de Blok et al., 2019).

Dados sobre as taxas de câncer de mama e endométrio em mulheres lésbicas e bissexuais são variáveis. Além de fumar, esse grupo tem taxas mais altas de obesidade, nuliparidade e hipertensão, fatores que podem contrinuir para neoplasias. Além disso, as MSGs são frequentemente menos propensas a fazer mamografia (McCune \& Imborek, 2018).

Certos comportamentos sexuais podem ainda colocar MSG em risco aumentado para cepas de papilomavírus humano (HPV) associadas ao câncer, incluindo: idade mais precoce na primeira relação sexual, mais experiências com parceiros e menor frequência de uso de preservativo durante a relação sexual. Além disso, parcela significativa de homens transgêneros não realizam rastreamento de câncer do colo do útero periodicamente (Ceres et al., 2018) Aumentar os esforços para intervenções como a vacinação contra o HPV nessa população e a triagem entre aqueles com maior risco pode ser benéfico.

É importante notar que a terapia com testosterona induz atrofia nos tecidos do colo do útero, o que resulta em frequentes resultados de Papanicolau insuficientes. O teste de HPV de alto risco (hrHPV) é importante na triagem dessa população, devido aos resultados de citologia não confiáveis e à maior possibilidade de auto coleta, o que pode melhorar a aceitação da triagem. Muitos países já utilizam o teste primário de hrHPV para rastreamento do câncer do colo do útero, apoiando ainda mais essa prática (Fontham et al., 2020; Seay et al., 2017; McDowell et al., 2017). De qualquer forma, os exames clínicos ainda devem ser incentivados, pois podem detectar condições pré-malignas/malignas que de outra forma não seriam detectadas apenas por citologia ou teste de hrHPV.

Muitos profissionais de saúde concluem incorretamente que as pacientes lésbicas não precisam de rastreamento do câncer do colo do útero porque têm baixo risco de câncer do colo do útero. Isso se baseia na suposição errônea de que a paciente não fez sexo com homens anteriormente (ACOG, 2021).

MSG em geral apresentam taxas mais altas de uso de cigarro/tabaco e uso de álcool, fatores de estilo de vida relacionados ao câncer importantes a serem considerados, associam-se a neoplasia cervical, vulvar e de ovário epitelial mucinoso. Lésbicas apresentam mais baixa paridade e obesidade, associados a maior risco de mama e de ovário, além de diabetes e doenças cardiovasculares (ACOG, 2021). Mais esforços são necessários para entender a prevalência, bem como os resultados do câncer nessa população. Estudos epidemiológicos ne grupo serão benéficos para identificar necessidades clínicas específicas e necessidades potenciais para triagem avançada ou precoce de doenças ginecológicas (Stenzel et al., 2020).

\section{ISTs}

Faz parte do cuidado em saúde sexual oferecer espaço seguro para que a pessoa possa conversar e buscar esclarecimentos. Durante a consulta, os profissionais devem questionar o tipo de relacionamento das pacientes, considerando identidades e práticas sexuais diversas (Moscheta et al., 2016). A recomendação de uso de métodos de barreira, por exemplo, precisa considerar a realidade das práticas sexuais de cada pessoa. Camisinhas podem ser utilizadas para qualquer tipo de penetração, por pênis, dedos ou objetos. Luvas e dedeiras também podem servir para a penetração por mãos, que devem ser mantidas limpas e com as unhas bem aparadas para evitar lesões da mucosa penetrada ou rupturas da barreira utilizada (McCune \& Imborek, 2018; São Paulo, 2020).

Infecções, incluindo vaginose bacteriana, candidíase, herpes e infecções por papilomavírus humano, podem ser contraídas por lésbicas. Mulheres bissexuais têm as maiores taxas de soropositividade para HIV em comparação com as lésbicas e mulheres heterossexuais. Práticas sexuais entre vulvas (como o tribadismo) também têm o potencial de transmitir ISTs. Métodos de barreira específicos para a prática de tribadismo ou para sexo oral em vulva são placas ou calcinhas com fundo de silicone ou látex resistentes, mas dificilmente são encontrados para comercialização e são pouco tolerados de modo geral. Filmes plásticos de uso culinário não devem ser utilizados por serem porosos e se romperem com facilidade. Uma alternativa viável é confeccionar uma placa de material seguro a partir de uma camisinha (interna ou externa): abrir o pacote da 
camisinha e desenrolá-la completamente, retirar o anel da extremidade, cortar ou rasgar a camisinha desde sua base e ao longo de todo seu comprimento, segurar a placa formada com ambas as mãos e mantê-la esticada para evitar o contato direto com a vulva (Pinto et al., 2005; Knight \&., 2017).

Homens transgêneros podem se identificar exclusivamente como gays, bissexuais, pansexuais ou assexuais, ou podem, às vezes, praticar sexo anal com pessoas cujo sexo biológico é masculino, como homens cisgêneros ou mulheres transgêneros e podem usar brinquedos sexuais compartilhados para penetração anal. Embora o exame de citologia anal juntamente com a triagem anal de HPV não sejam consensuais, tratam-se de testes de triagem comumente oferecido em programas especializados LGBTQ, especialmente para pessoas infectadas pelo HIV ou imunossuprimidas (Nisly et al., 2018; (Imborek et al., 2017).

É importante reconhecer que identidade de gênero é independente da orientação sexual e do comportamento sexual. As decisões sobre os testes de triagem de IST devem ser baseadas em uma história completa que inclui anatomia atual, comportamento sexual e outras práticas (Imborek et al., 2017).

\section{Planejamento Reprodutivo}

Como a sexualidade e o comportamento podem ser muito diversos entre pessoas de todos os gêneros, a prevenção de gravidez indesejada deve ser abordada para todas as pessoas que têm útero e ovários. Everett et al. (2018) identificaram que as mulheres lésbicas eram menos propensas a receberam aconselhamento contraceptivo e sobre o uso de preservativo em consultas relacionadas a ISTs, em comparação com mulheres heterossexuais. Deve ser lembrado que a maioria das mulheres lésbicas tem prática sexual com homem em algum momento de suas trajetórias, uma evidência da fluidez de suas práticas (Rufino et al., 2018)

Indivíduos transgênero parecem ter menores taxas de contracepção e rastreamento de câncer do que a população geral (Stewart et al., 2020; Imborek et al., 2017). Como a reposição de testosterona por si só não é um método confiável para prevenir a gravidez, deve-se considerar planejamento reprodutivo em indivíduos que fazem sexo vaginal regular ou ocasionalmente.

Os dispositivos intrauterinos não hormonais muitas vezes são preferidos, para evitar o efeito hormonal. No entanto, os métodos hormonais podem ser utilizados com segurança. Pode haver preocupações com relação aos efeitos associados aos métodos contraceptivos contendo estrogênio ou progesterona quanto a redução do tamanho do clitóris ou aumento das mamas ou mesmo reversão da masculinização. Embora isso normalmente não seja visto em pacientes que recebem doses adequadas de testosterona enquanto recebem formas hormonais de contracepção, uma discussão sensível e cuidadosa precisa ocorrer para abordar essas preocupações (Nisly et al., 2018).

O ginecologista deve também ser capaz de ajudar as pacientes a planejar a gravidez e alcançar concepção, além de fornecer encaminhamento para quaisquer opções de assistência à fertilidade necessárias, como preservação de fertilidade antes de hormonioterapia cruzada, barriga de aluguel e adoção, conforme indicado. MSGs frequentemente buscam essas informações on-line, o que muitas vezes perpetua a desinformação.

\section{Treinamento Profissional}

Considerando as barreiras ao cuidado vivenciadas por esses pacientes, é fundamental continuar as discussões sobre a redução do estigma das identidades sexuais e de gênero entre o campo da saúde e a população em geral. Atuar pelo conforto da pessoa inclui criar ambiente e sala de espera acolhedores, explicar o procedimento antes de realizá-lo, elucidar dúvidas, garantir o direito de ter uma pessoa acompanhante e compreender quais termos são mais bem aceitos pela pessoa para tratar de partes do seu corpo. Essas iniciativas terão como objetivo aumentar o conforto desses indivíduos no ambiente clínico, 
aumentar o conhecimento de necessidades específicas entre os profissionais de saúde e reduzir o potencial de violência e discriminação experimentado por indivíduos da comunidade transgênero. O respeito ao nome social entre transgêneros, por exemplo, gerou redução de $29 \%$ nas ideações suicidas e de 56\% de comportamentos suicidas entre jovens dos EUA (Russell et al., 2018; Rocon et al., 2016; Dendrinos et al., 2019; McCune \& Imborek, 2018).

A falta de conhecimento e de treinamento dos profissionais de saúde sobre como cuidar de especificidades desses grupos minoritários também contribui para esse distanciamento. Os currículos médicos apresentam lacunas no atendimento aos pacientes de MSG. O aumento do treinamento entre gestores e profissionais de saúde, além de melhorias gerais no sentido de compreender as barreiras ao acesso à saúde podem reduzir algumas disparidades. Pesquisas adicionais para triagem e vigilância do câncer nesta comunidade serão necessárias para entender quaisquer riscos adicionais potenciais e disparidades de sobrevivência experimentadas pelas MSG (Stenzel et al., 2020; Labanca et al., 2020).

Resultados dos exames laboratoriais e de imagem, por exemplo, podem mudar significativamente à medida que os pacientes recebem terapia hormonal. Dados normativos para exames laboratoriais e imagens radiológicas na população transgênero ainda não foram definidos. Alguns estudos preliminares mostram alterações comuns, como aumento nos níveis de hematócrito e hemoglobina em pacientes que recebem terapia com testosterona e alterações nos testes de função renal (Goldstein et al., 2017; Whitley \& Greene, 2017). O conhecimento dessas alterações permite monitorar e tranquilizar os pacientes.

Ainda há muito o que ser feito para a redução das situações de discriminação nos serviços de saúde. Em primeira análise, ao se sentir reconhecido, compreendido em sua autodeterminação de identidade de gênero e, principalmente, respeitado, o usuário sente-se confortável para acessar os serviços e usufruir de seus direitos em saúde, possibilitando a adesão ao tratamento e o prosseguimento nas consultas e tratamentos necessários (Silva et al., 2017; Mello et al., 2011).

\section{Considerações Finais}

Os profissionais de saúde devem se familiarizar com as necessidades únicas de atenção à saúde de pessoas que se identificam como homens transgêneros e mulheres homoafetivas, em especial o ginecologista. Diversos determinantes sociais podem influenciar o atendimento ginecológico das minorias sexuais e de gênero, podendo gerar disparidades de saúde nesses grupos marginalizados. Tais barreiras podem incluir componentes psicossociais, discriminação social, falta de acesso a cuidados adequados e apropriados e falhas na formação de profissionais de saúde.

A partir dos resultados desse estudo, é importante destacar a relevância de pesquisas futuras que ampliem essa temática e envolvam melhorias na gestão do sistema de saúde e de formação de médicos, em especial ginecologistas, além de meios de promover um atendimento que aborde as particularidades de homens transgêneros e mulheres homoafetivas. Dessa forma, poderá haver uma maior disseminação de conhecimentos e recomendações entre os profissionais de saúde, tendo como consequência a redução das hostilidades enfrentadas por essa parcela da população nos ambientes de atendimento, aumentando a adesão aos serviços de saúde e garantido o bem-estar.

\section{Referências}

American College of Obstetricians and Gynecologists' Committee on Gynecologic Practice; American College of Obstetricians and Gynecologists' Committee on Health Care for Underserved Women. (2021). Health Care for Transgender and Gender Diverse Individuals: ACOG Committee Opinion, Number 823. Obstet Gynecol, 137(3), e75-e88.

Arán, M., \& Murta, D. (2009). Do diagnóstico de transtorno de identidade de gênero às redescrições da experiência da transexualidade: uma reflexão sobre gênero, tecnologia e saúde. Physis: Revista de Saúde Coletiva, 19, 15-41.

Ávila, M. B. (2003). Direitos sexuais e reprodutivos: desafios para as políticas de saúde. Cadernos de Saúde Pública, 19, S465-S469. 
Bento. B. (2017). O que é transexualidade. Taubaté, São Paulo: Brasiliense.

Brasil. (2012). Ministério da Saúde. Carta dos direitos dos usuários da saúde. https://conselho.saude.gov.br/biblioteca/livros/Carta5.pdf.

Ceres, M., Quinn, G. P., Loscalzo, M., \& Rice, D. (2018). Cancer screening considerations and cancer screening uptake for lesbian, gay, bisexual, and transgender persons. In Seminars in oncology nursing (Vol. 34, No. 1, pp. 37-51). WB Saunders.

Chandra, A., Copen, C. E., \& Mosher, W. D. (2013). Sexual behavior, sexual attraction, and sexual identity in the United States: Data from the 2006-2010 National Survey of Family Growth. In International handbook on the demography of sexuality (pp. 45-66). Springer, Dordrecht.

Coleman, E., Bockting, W., Botzer, M., Cohen-Kettenis, P., DeCuypere, G., Feldman, J., ... \& Zucker, K. (2012). Standards of care for the health of transsexual, transgender, and gender-nonconforming people, version 7. International journal of transgenderism, 13(4), 165-232.

Costa, L. B. F., Rosa-e-Silva, A. C. J. D. S., Medeiros, S. F. D., Nacul, A. P., Carvalho, B. R. D., Benetti-Pinto, C. L., ... \& Maranhão, T. M. D. O. (2018). Recommendations for the use of testosterone in male transgender. Revista Brasileira de Ginecologia e Obstetrícia, 40, 275-280.

de Blok, C. J., Wiepjes, C. M., Nota, N. M., van Engelen, K., Adank, M. A., Dreijerink, K. M., ... \& den Heijer, M. (2019). Breast cancer risk in transgender people receiving hormone treatment: nationwide cohort study in the Netherlands. Bmj, 365.

De Roo, C., Tilleman, K., T’Sjoen, G., \& De Sutter, P. (2016). Fertility options in transgender people. International Review of Psychiatry, 28(1), 112-119.

Dendrinos, M. L., Budrys, N. M., \& Sangha, R. (2019). Addressing the Needs of Transgender Patients: How Gynecologists Can Partner in Their Care. Obstetrical \& gynecological survey, 74(1), 33-39.

Everett B.G., Higgins J.A., Haider S., Carpenter E. (2019). Do Sexual Minorities Receive Appropriate Sexual and Reproductive Health Care and Counseling? J Womens Health (Larchmt), 28(1), 53-62.

Fernandez, J., \& Tannock, L. (2016). Metabolic effects of hormone therapy in transgender patients. Endocrine Practice, 22(4), 383-388.

Fontham, E. T., Wolf, A. M., Church, T. R., Etzioni, R., Flowers, C. R., Herzig, A., ... \& Smith, R. A. (2020). Cervical cancer screening for individuals at average risk: 2020 guideline update from the American Cancer Society. CA: A Cancer Journal for Clinicians, 70(5), 321-346.

Goldstein, Z., Corneil, T. A., \& Greene, D. N. (2017). When gender identity doesn't equal sex recorded at birth: the role of the laboratory in providing effective healthcare to the transgender community. Clinical chemistry, 63(8), 1342-1352.

Gøtzsche, P. C., \& Jørgensen, K. J. (2013). Screening for breast cancer with mammography. Cochrane database of systematic reviews, (6).

Grimstad, F. W., Fowler, K. G., New, E. P., Ferrando, C. A., Pollard, R. R., Chapman, G., ... \& Gray, M. (2019). Uterine pathology in transmasculine persons on testosterone: a retrospective multicenter case series. American Journal of Obstetrics and Gynecology, 220(3), 257-e1.

Hembree, W. C., Cohen-Kettenis, P. T., Gooren, L., Hannema, S. E., Meyer, W. J., Murad, M. H., ... \& T’Sjoen, G. G. (2017). Endocrine treatment of genderdysphoric/gender-incongruent persons: an endocrine society clinical practice guideline. The Journal of Clinical Endocrinology \& Metabolism, 102(11), 38693903

Imborek K.L., Graf E.M., McCune K. (2017) Preventive Health for Transgender Men and Women. Semin Reprod Med, 35(5), 426-433.

Iwamoto, S. J., Grimstad, F., Irwig, M. S., \& Rothman, M. S. (2021). Routine Screening for Transgender and Gender Diverse Adults Taking GenderAffirming Hormone Therapy: a Narrative Review. Journal of General Internal Medicine, 1-10.

James, S., Herman, J., Rankin, S., Keisling, M., Mottet, L., \& Anafi, M. A. (2016). The report of the 2015 US transgender survey.

Jarin. (2019). The Ob/Gyn and the transgender patient. Curr Opin Obstet Gynecol, 31(5), 298-302.

Knight, D. A., \& Jarrett, D. (2017). Preventive health care for women who have sex with women. American family physician, 95(5), 314-321

Labanca, T., Mañero, I., \& Pannunzio, M. (2020). Transgender patients: considerations for routine gynecologic care and cancer screening. International Journal of Gynecologic Cancer, 30(12).

Lionço, T. (2009). Comprehensiveness and sexual diversity in the Brazilian norm in the process of health care for transsexuals: progress, dilemmas, challenges. Physis: Revista de Saúde Coletiva, 19(1), 43-63.

Lovison, R., Ascari, T. M., de Azambuja Zocche, D. A., Durand, M. K., \& Ascari, R. A. (2019). Travestis e transexuais: despindo as percepções acerca do acesso e assistência em saúde. Enfermagem em Foco, 10(5).

McDowell, M., Pardee, D. J., Peitzmeier, S., Reisner, S. L., Agénor, M., Alizaga, N., ... \& Potter, J. (2017). Cervical cancer screening preferences among trans-masculine individuals: patient-collected human papillomavirus vaginal swabs versus provider-administered pap tests. LGBT health, 4(4), $252-259$.

McCune K.C., Imborek K.L. (2018) Clinical Care of Lesbian and Bisexual Women for the Obstetrician Gynecologist. Clin Obstet Gynecol, 61(4), 663-673.

Mello, L., Perilo, M., Braz, C. A. D., \& Pedrosa, C. (2011). Políticas de saúde para lésbicas, gays, bissexuais, travestis e transexuais no Brasil: em busca de universalidade, integralidade e equidade. Sexualidad, Salud y Sociedad (Rio de Janeiro), 7-28.

Moscheta, M. S.; Fébole, D. S.; \& Anzolin, B. (2016). Visibilidade seletiva: a influência da heterossexualidade compulsória nos cuidados em saúde de homens gays e mulheres lésbicas e bissexuais. Saúde \& Transformação Social/Health \& Social Change, 7(3), 71-83.

Nisly, N. L., Imborek, K. L., Miller, M. L., Kaliszewski, S. D., Williams, R. M., \& Krasowski, M. D. (2018). Unique primary care needs of transgender and gender non-binary people. Clinical obstetrics and gynecology, 61(4), 674-686. 
Obedin-Maliver, J., \& de Haan, G. (2017). Gynecologic care for transgender adults. Current Obstetrics and Gynecology Reports, 6(2), 140-148.

Pinto, V. M., Tancredi, M. V., Neto, A. T., \& Buchalla, C. M. (2005). Sexually transmitted disease/HIV risk behaviour among women who have sex with women. Aids, 19, S64-S69.

Popadiuk, G. S., Oliveira, D. C., \& Signorelli, M. C. (2017). A Política Nacional de Saúde Integral de Lésbicas, Gays, Bissexuais e Transgêneros (LGBT) e o acesso ao processo transexualizador no Sistema Único de Saúde (SUS): avanços e desafios. Ciência \& Saúde Coletiva, 22, 1509-1520.

Puechl A.M., Russell K., Gray B.A. (2019). Care and Cancer Screening of the Transgender Population. J Womens Health (Larchmt), 28(6), 761-768.

Reis, T. (2018). Manual de Comunicação LGBTI+. https://www.grupodignidade.org.br/wp-content/uploads/2018/05/manual-comunicacao-LGBTI.pdf

Rocon, P. C., Rodrigues, A., Zamboni, J., \& Pedrini, M. D. (2016). Dificuldades vividas por pessoas trans no acesso ao Sistema Único de Saúde. Ciência \& Saúde Coletiva, 21, 2517-2526.

Rufino A.C., Madeiro A., Trinidad A., Santos R., Freitas I. (2018). Sexual practices and health care of women who have sex with women: 2013-2014. Epidemiol Serv Saude, 27(4), e2017499.

Russell, S. T., Pollitt, A. M., Li, G., \& Grossman, A. H. (2018). Chosen name use is linked to reduced depressive symptoms, suicidal ideation, and suicidal behavior among transgender youth. Journal of Adolescent Health, 63(4), 503-505.

São Paulo (2020). Secretaria Municipal da Saúde. Coordenação da Atenção Primária à Saúde. Protocolo para o atendimento de pessoas transexuais e travestis no município Paulo. https://www.prefeitura.sp.gov.br/cidade/secretarias/upload/saude/Protocolo_Saude_de_Transexuais_e_Travestis_SMS_Sao_Paulo_3_de_Julho_2020.pdf.

Seay, J., Ranck, A., Weiss, R., Salgado, C., Fein, L., \& Kobetz, E. (2017). Understanding transgender men's experiences with and preferences for cervical cancer screening: a rapid assessment survey. LGBT health, 4(4), 304-309.

Silva, L. K. M. D., Silva, A. L. M. A. D., Coelho, A. A., \& Martiniano, C. S. (2017). Uso do nome social no Sistema Único de Saúde: elementos para o debate sobre a assistência prestada a travestis e transexuais. Physis: Revista de Saúde Coletiva, 27, 835-846.

Souza, M. T. D., Silva, M. D. D., \& Carvalho, R. D. (2010). Revisão integrativa: o que é e como fazer. Einstein (São Paulo), 8, $102-106$.

Spizzirri, G., Eufrásio, R., Lima, M. C. P., de Carvalho Nunes, H. R., Kreukels, B. P., Steensma, T. D., \& Abdo, C. H. N. (2021). Proportion of people identified as transgender and non-binary gender in Brazil. Scientific reports, 11(1), 1-7.

Stenzel, A. E., Moysich, K. B., Ferrando, C. A., \& Starbuck, K. D. (2020). Clinical needs for transgender men in the gynecologic oncology setting. Gynecologic oncology.

Stewart, T., Lee, Y. A., \& Damiano, E. A. (2020). Do transgender and gender diverse individuals receive adequate gynecologic care? An analysis of a rural academic center. Transgender health, 5(1), 50-58.

Urban, R. R., Teng, N. N., \& Kapp, D. S. (2011). Gynecologic malignancies in female-to-male transgender patients: the need of original gender surveillance. American journal of obstetrics and gynecology, 204(5), e9-e12.

White Hughto, J. M., Rose, A. J., Pachankis, J. E., \& Reisner, S. L. (2017). Barriers to gender transition-related healthcare: Identifying underserved transgender adults in Massachusetts. Transgender Health, 2(1), 107-118.

Whitley, C. T., \& Greene, D. N. (2017). Transgender man being evaluated for a kidney transplant. Clinical chemistry, 63(11), $1680-1683$.

Wingo E., Ingraham N., Roberts S.C.M. (2018) Reproductive Health Care Priorities and Barriers to Effective Care for LGBTQ People Assigned Female at Birth: A Qualitative Study. Womens Health Issues, 28(4), 350-357.

Winter, S., Diamond, M., Green, J., Karasic, D., Reed, T., Whittle, S., \& Wylie, K. (2016). Transgender people: health at the margins of society. The Lancet, 388(10042), 390-400. 